\title{
A CRITIQUE ON INTERDISCIPLINARY QUANTUM SYSTEMS
}

\author{
Uthamacumaran, A. \\ a utham@live.concordia.ca \\ Concordia University, Montreal
}

\section{ABSTRACT}

Three cross-disciplinary branches of quantum science, namely that of: Quantum Chaos, Quantum Biology and Quantum Computation, are concisely addressed herein. The implications of these fields in the progression of science are emphasized. This critique is to be treated as a metacognition on currently contentious branches of science interwoven with the foundations of modern science: Quantum Mechanics, made accessible in layman terms to all systems thinkers.

\section{INTRODUCTION TO QUANTUM MECHANICS}

Quantum derives roots from the Latin word Quantus, referring to quantity (how much). The discovery that particles are discrete packets of energy with wave-like properties is inarguably our most successful scientific revolution to date, with repeated empirical confirmations. A quick glance at the photograph of the masterminds who pioneered this intellectual forest, at the 1927 Solvay Conference, will immediately seed forth ego dissolution. But what exactly is Quantum Mechanics (QM)? Most undergraduates in physics will think of D.J. Griffith's and J.J. Sakurai's classic textbooks on the formalism of QM or rather the Richard Feynman's lectures. Note we say quantum mechanics and not quantum dynamics. Quantum mechanics (QM) fundamentally governs everything. The information age sprung from this foundation; for instance, recall the transistors in our cellphones and computers. Likewise, QM has also become the destroyer of worlds; the nuclear bombs and its victims have many stories to tell with this regard. Wherever there is matter (atoms), there is QM. Both QM and chaos are bizarre, puzzling, and counter intuitive. To merge the two pillars, Merlin's beard - let us hold this ambitious thought (system) for now.

The birth of QM began with the problem of blackbody radiation. Classical models failed to describe its energy spectrum. In 1900, Max Planck, often denoted as the father of Quantum Mechanics, corrected the intensity spectral distribution by taking into account that electromagnetic radiation can only be emitted or absorbed in discrete quanta (packets) of energy. These discrete packets were then demonstrated by Albert Einstein and Satyendra Bose as the basis of the photoelectric effect; what is today referred to as photons. Thus, QM tells us all quantifiable information characteristic to a system, i.e., energy, frequency, and vibrations, are quantized (come in discrete packets). The laws of quantum mechanics are very strange and quite nostalgic to the faint hearts of men; even the pioneers of this theory have either gone bonkers or surrendered to its incomprehensibility. Then imagine how much humility one must entail to grasp the marriage between quantum mechanics and nonlinear dynamics? More importantly, does it even make sense (ignoring the fact that neither do by themselves alone)? Perhaps this is a topic best dealt with the mathematicians but let us transiently explore these fundamental barriers in scientific thinking for intuition.

\section{QUANTUM CHAOS: THE PHANTOM BRIDGE}

The problem of quantum chaos is within the formal structure of the two branches it consists of themselves. Quantum mechanics assumedly pertains to small systems such as atoms and molecules; the behaviors of which appear like ripples on a pond due to wave-particle duality. Whereas, chaos theory deals with ODEs (ordinary differential equations) exhibiting sensitive dependence to initial conditions 
and perturbations. On the contrary, QM mainly deals with linear partial differential equations such as the Schrödinger wave equation, to which there are ordered, assumed solutions satisfying linearity and thereby, the superposition principle. Chaotic dynamics deals with nonlinearity and the solutions are either intractable or unpredictable, often requiring the exhaustive use of computational algorithms and heuristics to infer approximate numerical solutions. Chaos causes eigenfunctions of a quantum system to rapidly oscillate in all directions often resulting in non-integrability (1). Most models of quantum chaos currently in practice deal with simple mathematical models such as the kicked rotor, quantum billiards in a stadium, etc. to which the solutions are derived using semi-classical approaches. Many mathematical tools in this branch are used to study complex many body-systems. For example, Random Matrix Theory was developed by Wigner, Dyson, Mehta et al. originally intended to relieve the perplexity of spectra of complex nuclei, and later was repurposed in the spectra of all chaotic systems (2). Strict definitions of quantum chaos remain controversial and ambiguous, hence the incentive to this piece.

The current study of quantum chaos is often equated with a refined derivative of semi-classical physics. Let us go back to the Bohr Hydrogen atom, which consists of quantized periodic orbits (stationary states) reminiscent of the planetary model, denoting the allowed "energy levels" the electron can occupy. However, these quantization conditions do not apply to more complicated systems. As the many-body quantum system reaches a certain level of complexity (at times, with nearly a few atoms), mean-field theory and similar statistical approaches are required. Schrödinger resolved the problem of how an electron moving around a nucleus can be moving and stationary simultaneously using his linear wave equation, but at the cost of losing the notions of classical mechanics (3). Thus, classicity is regained in the description of (many-body) quantum systems only as one approaches the semi-classical limit, where Planck's constant approaches zero. Most of what is today known as the study of quantum chaos refers to the special case of semi-classical systems where the classical Hamiltonian counterpart is chaotic (suggestive of an unpredictable dynamical system) (3). Quantum Chaos, in its formal mathematical study, thus refers to the quantization of classically chaotic Hamiltonian systems, rather than the emergence of chaos in quantum systems.

Some may argue, Quantum Chaos may pertain to how quantum vacuum fluctuations give rise to the emergent properties and evolution of all that is enclosed within the cosmos (i.e., cosmic inflation) (4). The vacuum is then to be treated in reminiscence of a viscous fluid undergoing turbulent fluctuations from which all information dynamics manifest (4). This can be advocated as a form of fine-tuning (anthropic) principle guiding the Quantum Evolution Hypothesis (QEH), i.e., that quantum vacuum fluctuations direct the emergence and time-evolution of natural systems, including biological systems. Whether one chooses to call these fluctuations as vacuum noise (randomness) or quantum chaos is then subjected to personalized opinions and controversy; it is a fuzzy regime of investigation currently lacking experimental methods. The QEH brings to our attention another widely debated, emerging discipline of thought: Quantum Biology.

\section{QUANTUM BIOLOGY: A COMPLICATED PARADOX}

Traditionally, quantum mechanics was limited to systems with low degrees of freedom and minimized thermal fluctuations. However, the emerging field of quantum biology asserts quantum phase transitions may help explain complex systems that are not fully defined by classical physics. Although alternate interpretations of quantum mechanics such as those pertaining to Madelung's hydrodynamics and de Broglie-Bohm theory are better in support of macroscopic quantum effects, quantum biology 
remains relatively a new field severely lacking experimental support. As such, it is subjected to the discontent and frustration of many.

Quantum biology asserts, certain biochemical systems such as protein complexes in the photosynthetic chain exhibit extended quantum coherences to nonlocally direct energy transfer (i.e., the energy transfer is best defined as a superposition of waves) $(5,6)$. However, while some papers support this, others claim these inter-exciton coherences are too short lived to have any functional significance. Again, it must be stressed there is a lack of experimental evidence and meta-analyses in this stream of thought, and more importantly a lack of sense-making (understanding) of existing results.

The energy production and transfer in biosystems pertains to open (nonequilibrium) thermodynamics. Hence, it is quite paradoxical to think about how quantum mechanics manifests in thermodynamical systems. That is, macroscopic structures and molecules are highly unlikely to tunnel through an energy barrier (in the order of $k_{B} T$, where $k_{B}$ is the Boltzmann constant and $\mathrm{T}$ is the thermodynamic temperature). Thermodynamics deals with many orders of magnitude (i.e., in the range of Avogadro's numbers) whereas quantum mechanics often deals with sub-atomic if not few atomic scale structures. Thence, emerges the paradox of quantum biology. However, still many nano-mesoscopic systems in soft matter physics are increasingly exhibiting macroscopic quantum effects as emergent phenomena. For example, enzymatic tunneling and the associated changes in protein conformations may involve quantum tunneling. With quantum tunneling, the transition-state energy barriers are bypassed $(7,8)$. The quantum coherence of collective vibrational modes in proteins are suggested to withstand thermal fluctuations allowing such emergent behaviors. Classical rate laws then become inadequate descriptions of the chemical kinetics in certain enzymatic processes (7). Furthermore, quantum effects such as proton tunneling between base pairs have been established as a promoter of mutagenesis (9). Although fundamentally everything is quantum-mechanical, quantum biology asserts that macroscopic quantum effects such as nonlocality and quantum coherence for extended time scales exist in many-body (bio)systems, further indicating a scaling-problem in systems science.

Furthermore, the transition from quantum to classical chaos in such systems has been suggestive of power-law behaviors (10). Power law decays are proposed to be able to explain the extended quantum coherence and quantum transport observed in the photosynthetic energy transfer of chromophores at ambient temperature (10). Power laws are signatures for the emergence of self-organization in many edges of chaos phenomena such as the critical fluctuations of phase-transitions, cell state bifurcations, scale-free networks and many biologically relevant nonequilibrium processes, as well. Hence, quantum biology, although paradoxical and seemingly implausible, seems to suggest there are certain information processes unfolded in nature which necessitates the use of quantum mechanics for a better description. This brings us to a final remark of this essay, the dawn of Quantum Computing, another disputed branch of interdisciplinary systems science foreshadowed to be the next revolution in scientific progression.

\section{QUANTUM COMPUTATION: THE PANDORA'S BOX}

Quantum computing is a highly controversial field, for it asks the following million-dollar question: Will quantum computation solve complexity problems (e.g., NP-class problems) without heuristics or numerical approximation methods? Quantum supremacy is the alleged claim that quantum computers can perform certain computational tasks or solve computational problems exponentially faster on a quantum processor (i.e., pertaining to qubits) than on a classical processor in a feasible amount of time 
(11). While a lower time complexity than classical algorithms is implied, whether there are any computability advantages can only be verified in time (11). The transdisciplinary marriage between quantum computation and medicine is further believed to be at the dawn of optimal Al assisted medical diagnosis and clinical decision making. The implications of such unions within the emerging branch of computational medicine is beyond the scope of this critique.

To demonstrate the confounding implications of quantum computing consider the following results. Recent experiments on IBM's Q-processor show there's protection of quantum information in a qubit system due to the many entanglements it generates throughout its time-evolution (12). As demonstrated by the study, time-travelling to the initial state of the qubit system to perturb its initial conditions prevents changes in the preserved present, denoting some form of hysteresis. The quantum correlations permitted local information to be essentially restored even with damaged information as opposed to initiating a butterfly effect that cascades to an alternate present (12). Of course, the Copenhagen interpretation insists the act of measurement itself can prevent such scenarios from happening and, the step by step time-transition of the quantum information system (dynamics) are untraceable. That is, keep in mind still, the quantum system in this study was perturbed postmeasurement.

We live in exciting times in the advent of science. Tech pythons like IBM and Google are now racing to commercialize quantum computers yet progress in error correcting codes in quantum computing remain relatively new for the time being to fully maximize the purported benefits. What was once believed to be science fiction alike the works of Isaac Asimov, transhumanist fantasies and cyberpunk movies, is now becoming reality in the invasive frontier of Artificial Intelligence (Al).

The anticipation of whether quantum computing will pave solutions to complexity problems makes this a hot topic worth the buzz of transdisciplinary scientists and cyberneticians. However, as demonstrated here, the problem of quantum chaos still imposes limitations in the ability of machine intelligence, even if they consist of quantum computing systems, in predicting causal information dynamics due to the blur in the notion of quantum chaos. Perhaps this may be the barrier for machine intelligence to overcome for true meaning-making processes and data contextualization. Computational physics demonstrates that even simple systems in software space can exhibit undecidability, intractability, computational irreducibility and non-analyticity, notions which cannot be understood from either or, but only by the mixing of concepts from both streams: Quantum and Chaos.

\section{FOOD FOR THOUGHT AND SOPHIA}

Here are a few concluding remarks or rather a philosophical ramble on systems thinking to put this tea party to rest. This transient critique is also a self-reflection and meta-cognition of my scientific thinking in time, post-training in a basic physics background. These thoughts have been unfolded in a whimsical tone herein to stipulate the playful minds one must embrace in attempting to reconcile such complex problems, alike children, and similar intention-seekers.

When encountering the above-discussed topics, think of science as a dynamic process, constantly being revised and unfolding. There must be strong theoretical and experimental results layered on top of each other while holding Popper's principle of falsification at heart for scientific results to hold validity. Repeated verification of scientific results is critical for scientific evolution. For example, it was assumed until very recently the microbiome consists of nearly $90 \%$ of the human body. However, revised 
estimates suggest it may be a near equal ratio to human cells. This pinpoints how a lack of repeating empirical findings hinders systems science, and more importantly it reiterates science is dynamic.

Think of how Newton's classical mechanics reigned for some time with the notion of absolute motion until swept by Einstein's relativity, or rather how the plum-pudding model eventually transformed into the Bohr atom (still a simplification), in time. Scientific models, no matter how refined they may currently seem, only reveal partial information (if not very little) or a distortion about the undivided whole. A clear example to illustrate this, in modern science, is the art of machine learning; the details of how these algorithms perform pattern recognition and their interpretation (meaning-making) still remain a mystery (e.g., the black box in Deep Learning networks). There are then fundamental limitations on what is knowable or verifiable whether it be within the logical structure of axiomatic rules (Gödel's incompleteness theorems), the limitation of algorithmic computation (i.e., K-complexity) or the quantum indeterminacy of physical measurements imposed by the Heisenberg uncertainty principle, to name a few.

While the practice of science is very disciplined and requires high virtues, there are historically repeated accounts where the masses of the crowd were disproven by rebellious scientists; the Renaissance (rebirth) and the scientific revolution it led is a testimony to this statement. There are also historical accounts of pioneers who had been suppressed and rejected by the crowds to the point of their suicide. Ludwig Boltzmann, the father of statistical mechanics, is a tragic example. Science is very human and personal, through the kaleidoscope of history.

Lastly, there are cases of fringe science which hinder scientific progress the most and raise controversy to the above-discussed systems (thoughts). The proponents of these fringe sciences may be reputable scientists themselves. For example, although supported by some pioneers and Nobel Laureates such as Jacques Beneviste, Luc Montagnier and Brian David Josephson, the claims of 'water memory' are labelled as borderline mumbo jumbo (13). These scientists have now become marginalized and scrutinized for their unsupported claims. For instance, Linus Pauling, a Nobel laureate known for his pioneering works in chemistry (and QM), falsely purported the effects of ascorbic acid (vitamin C) as a cure for cancer. These cringe sciences are often interconnected to the above-discussed topics as plausible mechanisms causing their validity to further diminish.

To conclude, keep in mind science is still very human, for it is the humans that conduct, develop, polish, advocate and nurture the science. Therefore, science still adheres to biases, the elimination of which is essential to taste a greater part of the collective whole. Remain skeptic but also curious alike children, innate systems scientists.

"Space is not empty. It is full, a plenum as opposed to a vacuum, and is the ground for the existence of everything, including ourselves. The universe is not separate from this cosmic sea of energy."- David Bohm

\section{REFERENCES}

1) Stöckmann, H-J., Quantum Chaos: An introduction (Cambridge University Press, 1999)

2) Gutzwiller, M. Quantum Chaos. Scholarpedia, 2(12):3146 (2007) 
3) Zelditch, S. Mathematics of Quantum chaos in 2019. Notices of the American Mathematical Society. Vol 66(9). Pp 1412-1422 (2019)

4) Davies, P.C.W. Quantum vacuum noise in physics and cosmology. Chaos. Vol. 11(3) pp. 539-547 (2001)

5) Marias, A. et al., The future of quantum biology. J of the Royal Soc. Interface (2018)

6) Engel, G. S. et al. Evidence for wavelike energy transfer through quantum coherence in photosynthetic systems. Nature. 446 (7137), 782-786. (2007)

7) Lambert, N. et al. Quantum Biology. Nature Phys. 9:10-18 (2012)

8) Marletto, C. et al., Entanglement between living bacteria and quantized light witnessed by Rabi splitting. J Phys Commun. 2:101001 (2018)

9) Löwdin, P.-O., Proton tunneling in DNA and its biological implications. Rev. Mod. Phys. 35 (3), 725-732 (1963)

10) Vattay, G. et al. Quantum biology on the Edge of Quantum Chaos. PLoS One. 9(3): e89017 (2014)

11) Arute, F. et al., Quantum supremacy using a programmable superconducting processor. Nature volume 574, pp. 505-510 (2019)

12) Yan, B., \& Sinitsyn, N. A. Recovery of Damaged Information and the Out-of-Time-Ordered Correlators. Physical Review Letters, 125(4) (2020)

13) Davenas, E. et al., Human basophil degranulation triggered by very dilute antiserum against IgE. Nature vol. 333, pp. 816-818(1988) 